\title{
Cardiovascular MRI of the pericardium: A case review of the anatomy, scan protocols and pathology of the pericardium
}

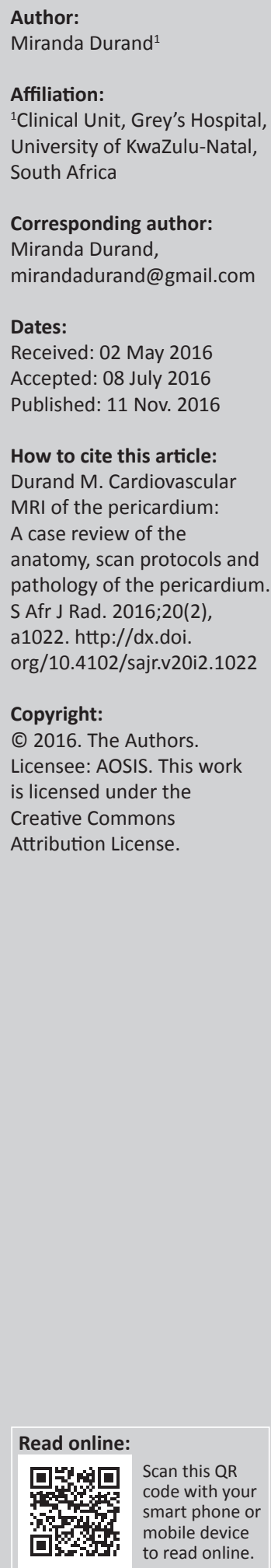

\begin{abstract}
The aim of this article was to present a case based review of the anatomy, scan protocols and pathology of the pericardium. Cardiovascular magnetic resonance imaging provides excellent anatomic depiction of the pericardium, vital information on myocardial infiltration and characterisation of mass lesions and pericardial effusions. It adds valuable information in the assessment of complicated pericardial disease.
\end{abstract}

\section{Introduction}

Cardiovascular magnetic resonance imaging (CMR) is emerging as an important modality for the assessment of pericardial disease. It has the advantage of providing excellent anatomic depiction of the pericardium; cine images provide vital information on local myocardium infiltration; and the different MRI sequence options help to characterise mass lesions and pericardial effusions. The aim of this article is to use clinical cases as a basis from which to review and discuss the anatomy, scan protocols and pathology of the pericardium.

\section{Case 1}

A 19-year-old man with a repaired left Bochdalek congenital diaphragmatic hernia, subsequently presented with right atrial and ventricular enlargement on echocardiography secondary to left pulmonary artery hypoplasia. The patient was sent for CMR to assess right ventricular function and size; the scan also demonstrated an incidental absent pericardium.

The axial cine steady-state free precession (SSFP) images demonstrated the left upper lobe of the lung herniating into the potential space between the aorta and the main pulmonary artery (Figure 1a). In this region, the pericardium is normally seen as a bridge between the aorta and the main pulmonary artery (Figure $1 b$ ). In cases of absent pericardium, the heart is also often more horizontally orientated and displaced to the left (Figure 2a). At the level of the atrioventricular groove, the pericardium that is normally seen as a thin line lateral to the right coronary artery (Figure 2b), is absent in this patient (Figure 2a).

\section{Diagnosis: Absent pericardium}

The pericardium can be partially or completely absent. Partially absent pericardium is more common than a complete absence. It is more common for the left pericardium to be absent in the setting of partial absent pericardium than the right. Approximately a third of all absent pericardium is associated with other disorders such as tetralogy of Fallot, patent ductus arteriosus and atrial septal defects. ${ }^{1}$

\section{Pericardium anatomy}

The pericardium is a flask-like structure covering the heart and origin of the great arteries. It consists of the visceral and parietal layers, with a potential space between these two layers that can contain up to $15 \mathrm{ml}-50 \mathrm{ml}$ of fluid. The pericardium acts as a barrier against infection and inflammation, reduces friction between the heart and the mediastinal structures, and prevents excessive dilatation of the heart chambers. ${ }^{2}$ The normal pericardium measures less than $2 \mathrm{~mm}$ (Figure 2b) whilst a pericardial thickness of more than $4 \mathrm{~mm}$ indicates pericardial disease. ${ }^{3}$ A pericardial thickness of $3 \mathrm{~mm}-4 \mathrm{~mm}$ is equivocal and should be assessed in the setting of the clinical presentation of the patient. 

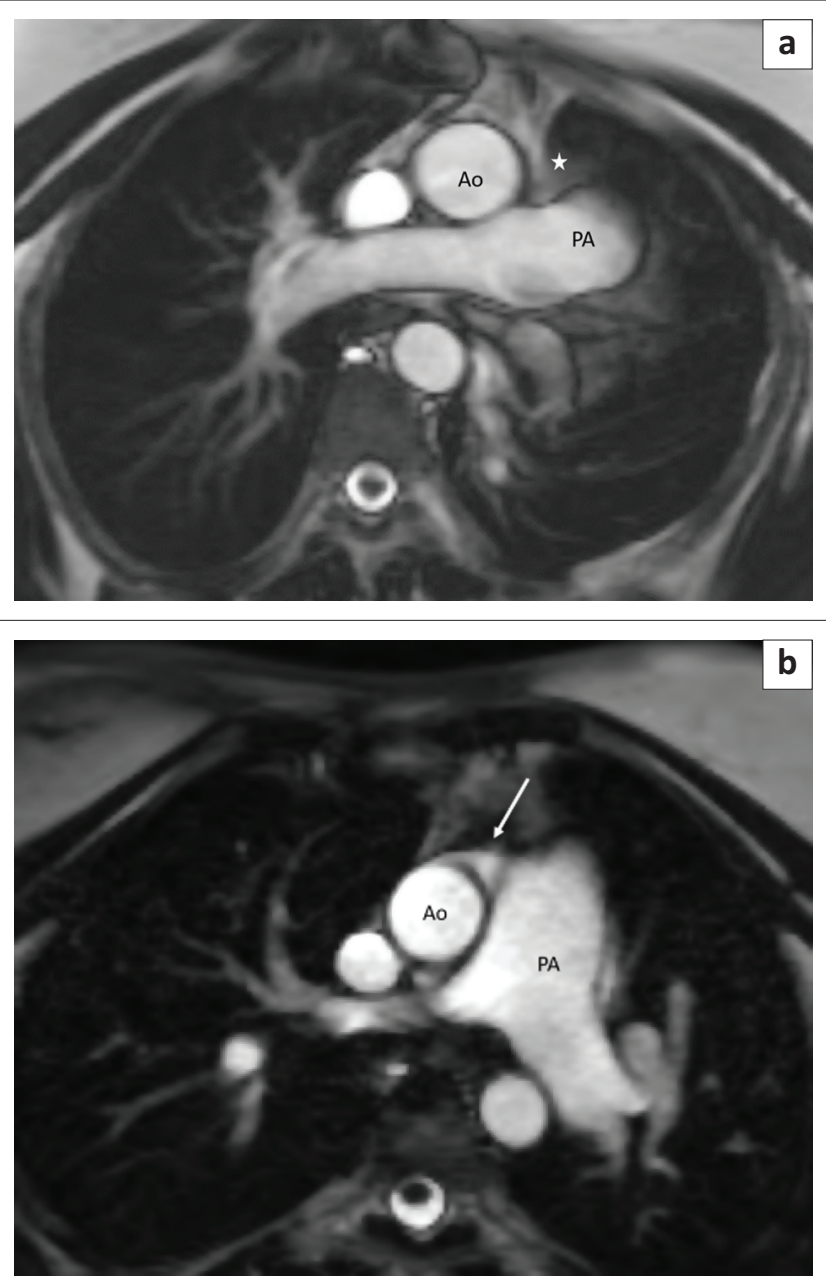

FIGURE 1: A 19-year-old man with absent pericardium. The axial SSFP image (a) demonstrates left lung $(*)$ herniating into the space between the aorta (Ao) and main pulmonary artery (PA). Normally (b) the pericardium is seen as a thin grey line (white arrow) bridging the space between the aorta $(\mathrm{Ao})$ and pulmonary artery (PA).

\section{Case 2}

A 54-year-old woman presented to the dermatology clinic with cutaneous papules. Biopsy of these lesions demonstrated non-caseating granulomas in keeping with cutaneous sarcoidosis. During the work-up of the patient, the echocardiography demonstrated a small pericardial effusion. A CMR was requested to assess for myocardial involvement and functional assessment.

CMR demonstrated a small pericardial effusion and pericardial thickening (Figure 3), with the pericardium measuring $4 \mathrm{~mm}$; this is often best demonstrated on the black blood sequence. The late gadolinium enhancement also demonstrated avid pericardial enhancement (Figure 4) in keeping with pericarditis. The patient did not have a pericardial tap or biopsy to confirm the diagnosis, but in light of the other clinical findings and absence of acute symptoms, the diagnosis of sarcoidosis pericarditis was made.

This patient did not demonstrate any myocardial thickening or abnormal myocardium enhancement to suggest myocardial involvement.
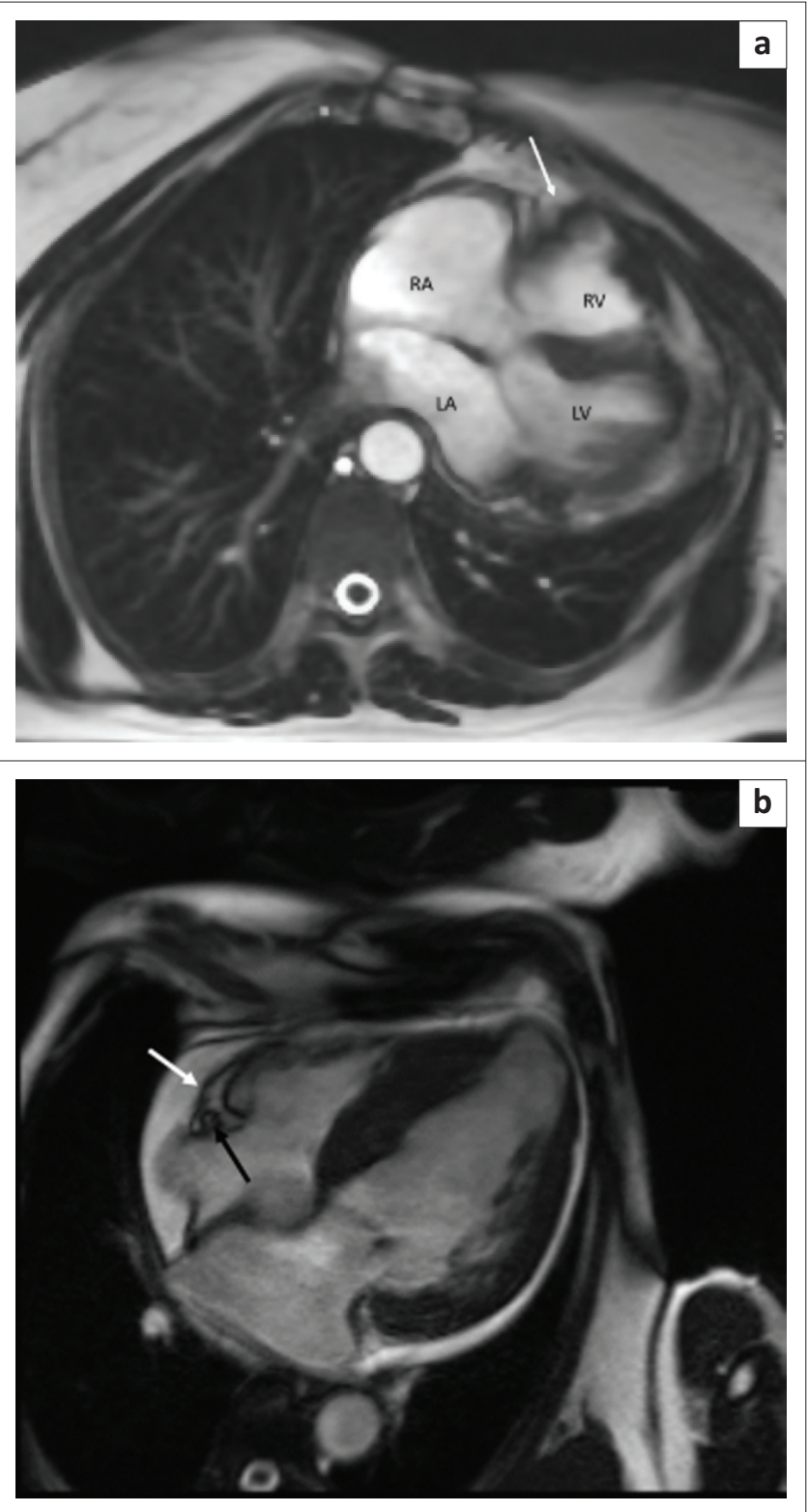

RA, right atrium; RV, right ventricle; LA, left atrium; LV, left ventricle.

FIGURE 2: A 19-year-old man with absent pericardium. Axial SSFP image (a) demonstrates a horizontally orientated heart with absence of the pericardium across the atrioventricular (AV) groove (white arrow). Normally (b) the pericardium is seen as a thin grey line crossing the AV groove (white arrow) lateral to the right coronary artery (black arrow).

\section{Diagnosis: Sarcoidosis pericarditis}

Pericardial involvement in sarcoidosis may occur with or without associated myocardial involvement. Up to $20 \%$ of patients with sarcoidosis will also have asymptomatic pericardial effusions. ${ }^{4}$ However, pericardial sarcoidosis can rarely present as a symptomatic pericardial effusion with or without associated tamponade or as a chronic constrictive pericarditis. In the absence of myocardial involvement, most of these patients have a good prognosis. ${ }^{5}$

\section{The role of CMR imaging in pericarditis}

Not all patients with acute pericarditis need to undergo a CMR investigation - most cases with classical clinical findings can accurately be assessed and treated with basic investigations. 


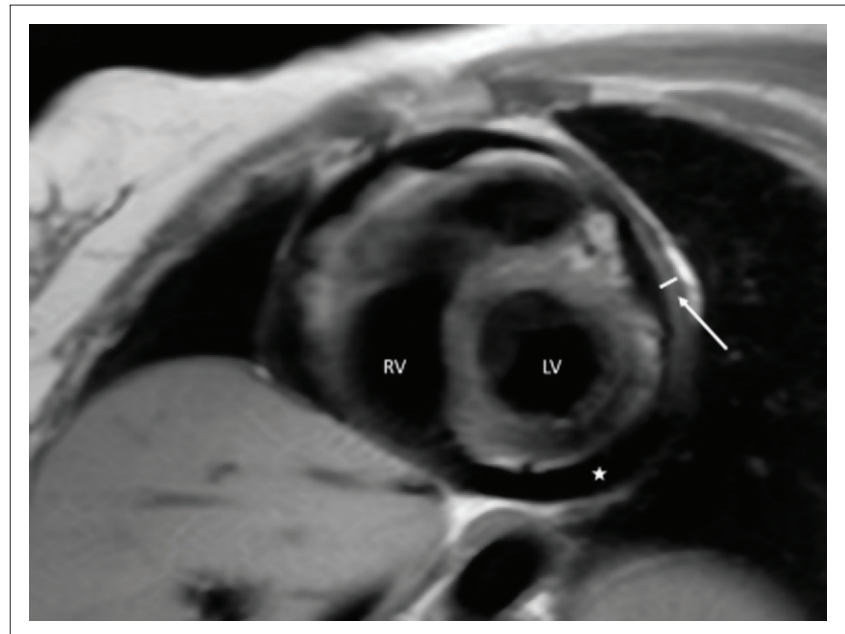

$\mathrm{RV}$, right ventricle; LV, left ventricle.

FIGURE 3: A 54-year-old woman with biopsy-proven cutaneous sarcoidosis. The black blood image in a short-axis orientation demonstrates a small pericardial effusion $(*)$ as well as thickening (white arrow) of the pericardium, with the pericardium measuring $4 \mathrm{~mm}$.

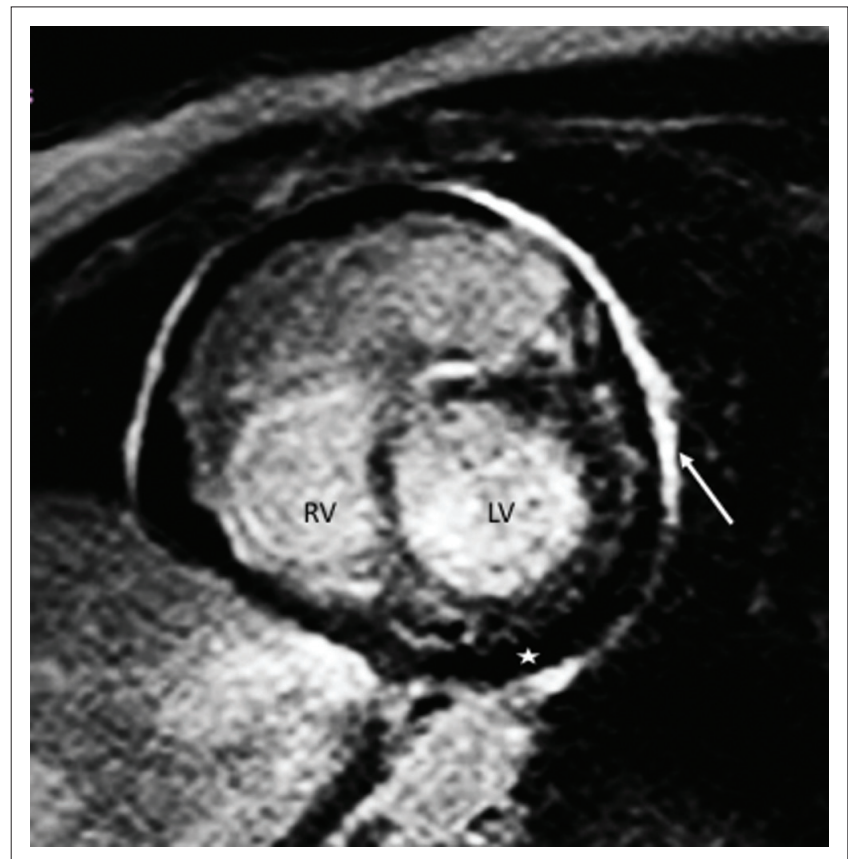

$\mathrm{RV}$, right ventricle; LV, left ventricle.

FIGURE 4: A 54-year-old woman with biopsy-proven cutaneous sarcoidosis. The late gadolinium enhancement image in the short-axis orientation demonstrates the pericardial effusion $(*)$ as well as avid pericardial enhancement (white arrow).

The role of CMR is to further assess those patients with atypical presentations, therapy failure, and those with a complicated course, with chronic or recurrent pericarditis, with or without constriction. In patients with associated diseases, such as sarcoidosis, or penetrating injury, CMR can also supplement computed tomography imaging to confirm the diagnosis, identify associated pathology and assess the influence on cardiac output and function. ${ }^{6}$

\section{Case 3}

A 62-year-old man with end-stage renal failure, on renal dialysis for the past 2 years, was sent for a CMR assessment for constrictive pericarditis after an echocardiogram demonstrated mildly dilated left and right atria.

The CMR demonstrated pericardial thickening with loculated pericardial effusions as well as hyperintensity of the pericardium on the short tau inversion recovery (STIR) images (Figure 5). The free-breathing cine SSFP images demonstrated 'septal bounce', confirming constrictive pericarditis (Figures 6 and 7).

\section{Diagnosis: Constrictive pericarditis}

Constrictive pericarditis is often idiopathic or viral but, in South Africa, a common cause is scarring from tuberculous pericarditis. Other causes also include previous surgery, radiation and malignancy. Rarely, end-stage renal disease (ESRD) can also result in constrictive pericarditis, ${ }^{6,7}$ although the more common manifestations of pericardial involvement of ESRD are acute uraemic or dialysis pericarditis. ${ }^{7}$ Cases of rapidly progressive pericardial calcification and calcified pericardial effusion as part of extra-skeletal calcification in ESRD owing to hyperparathyroidism have also been described. ${ }^{8}$

\section{Physiology of the 'septal bounce' on free- breathing steady-state free precession images}

'Septal bounce' is the characteristic septal movement seen in constrictive pericarditis and is used to differentiate constrictive pericarditis from restrictive cardiomyopathy. The 'septal bounce' occurs as a result of fixed pericardial volume secondary to pericardial fibrosis or calcification. This causes interventricular volume dependence, meaning that increased volume in the one ventricle causes a decrease in the volume in the adjacent ventricle.

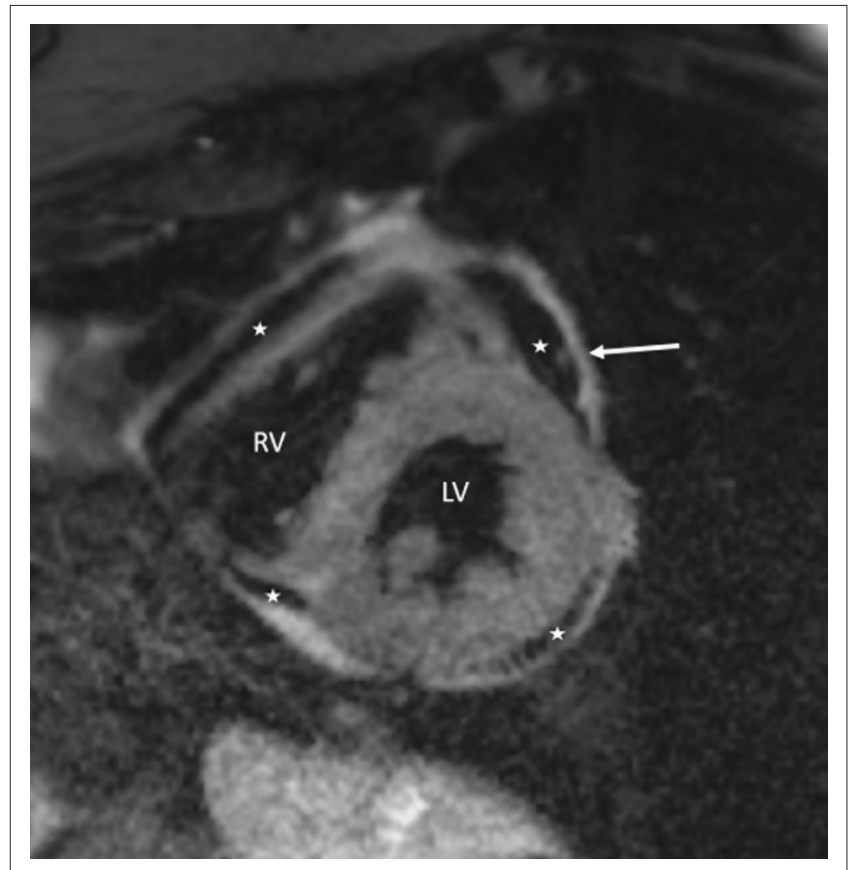

RV, right ventricle; LV, left ventricle.

FIGURE 5: A 62-year-old man with constrictive pericarditis. The short-axis STIR image demonstrates a loculated pericardial effusion $(*)$ and thickened hyperintense pericardium (white arrow). 


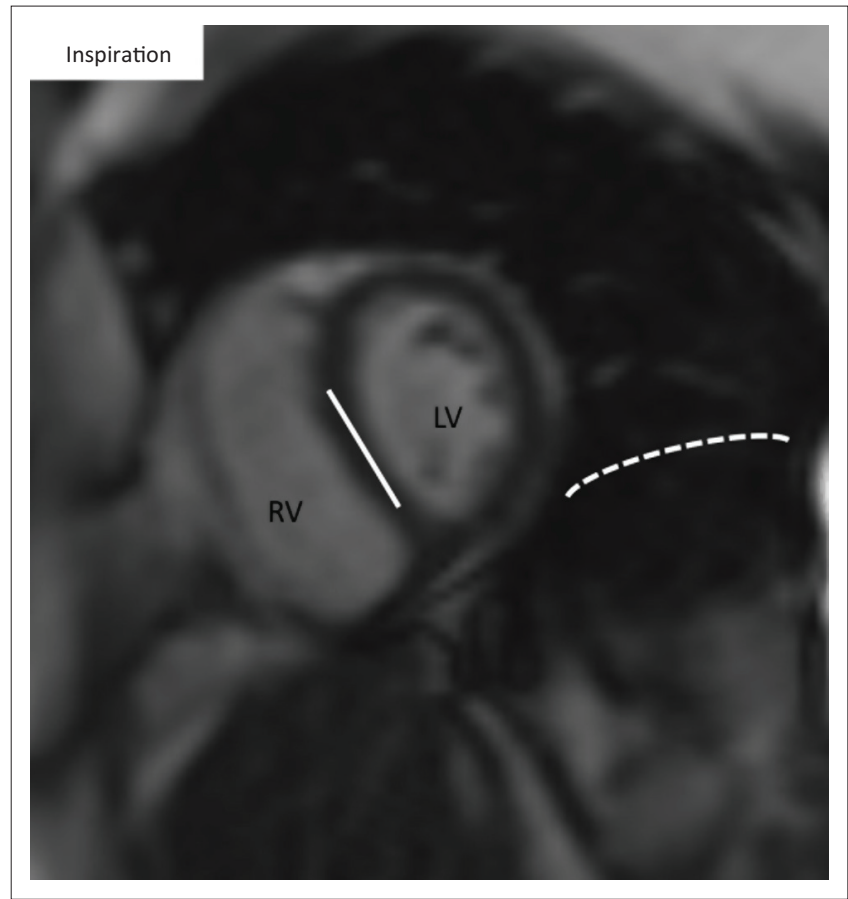

$\mathrm{RV}$, right ventricle; $\mathrm{LV}$, left ventricle.

FIGURE 6: A 62-year-old man with constrictive pericarditis. Free-breathing cine SSFP image at the mid-ventricular level on the short-axis view demonstrates flattening (solid line) of the interventricular septum during inspiration when the diaphragm is flat (dotted line).

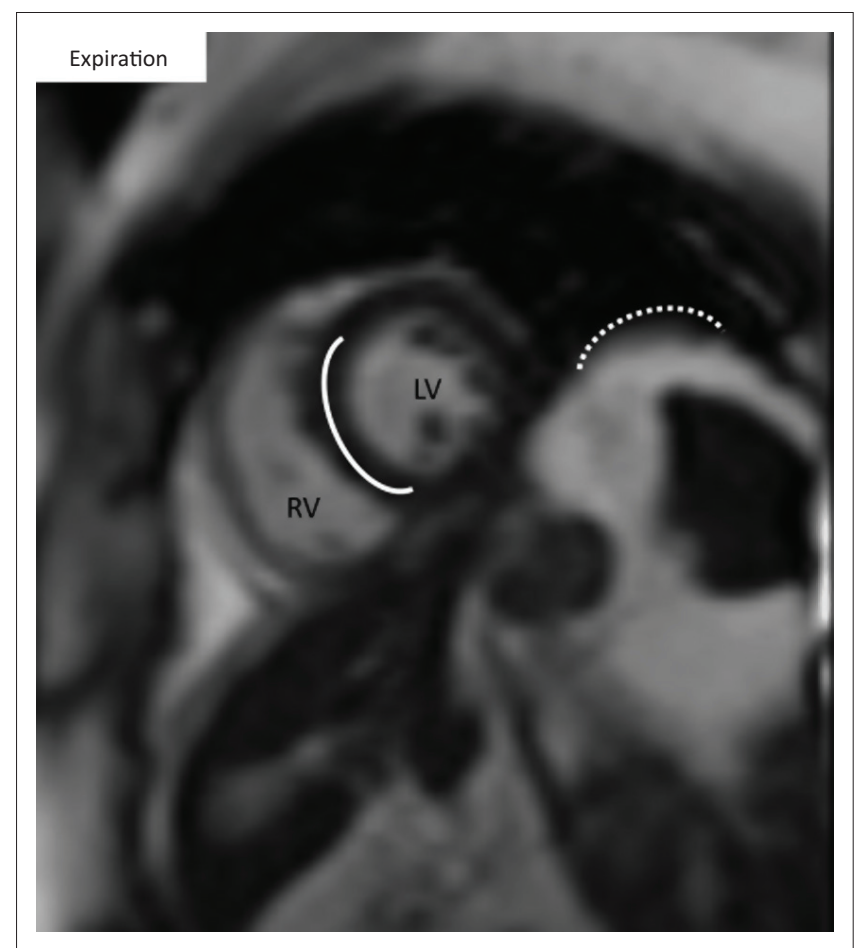

$\mathrm{RV}$, right ventricle; LV, left ventricle.

FIGURE 7: A 62-year-old man with constrictive pericarditis. Free-breathing cine SSFP image at the mid-ventricular level on the short-axis view demonstrates bulging (solid line) of the interventricular septum during expiration when the diaphragm is rounded (dotted line).

The filling of the right ventricle starts slightly earlier than that of the left ventricle and, with inspiration, there is an increase in the venous return to the right atrium and right ventricle, increasing the pressure in the right ventricle. The change in pressure results in a leftwards motion of the septum towards the left ventricle, flattening the normal curvature of the interventricular septum (Figure 6).

During expiration, there is decreased venous return to the right ventricle, and the pressure in the left ventricle increases relative to that of the right ventricle. This differential causes the septum to move towards the right ventricle reassuming its normal curvature (Figure 7). On the free-breathing SSFP images, these physiological changes can be visualised and accentuated by asking the patient to take in a deep breath during the acquisition of the short-axis cine images. ${ }^{9}$

The 'septal bounce' is very sensitive for constrictive pericarditis but not very specific. The 'septal bounce' has also been seen in other conditions such as cardiac tamponade, pulmonary arterial hypertension, left bundle branch block and right ventricular pacing. ${ }^{9}$

\section{Case 4}

A 33-year-old man with a 4-week history of chest pain had an echocardiogram as part of his work-up, which demonstrated a large mass adjacent to the right ventricle. The patient was sent for CMR assessment of the mass for further surgical planning. The CMR demonstrated a large hyper-intense mass adjacent to the right ventricle on the axial black blood images (Figure 8). The lesion was composed of fat signal intensity on all the sequences and demonstrated no enhancement on the late enhancement images. The axial cine SSFP images demonstrated in most regions a clear plane of separation between the mass and the myocardium of the right ventricle (Figure 9a), best seen during systole. However, there was a point on the cine SSFP images where the right ventricular myocardium did not pull away from the lesion during systole (Figure 9b) and a small area of myocardial infiltration with extension of the lesion into the right ventricle was demonstrated.

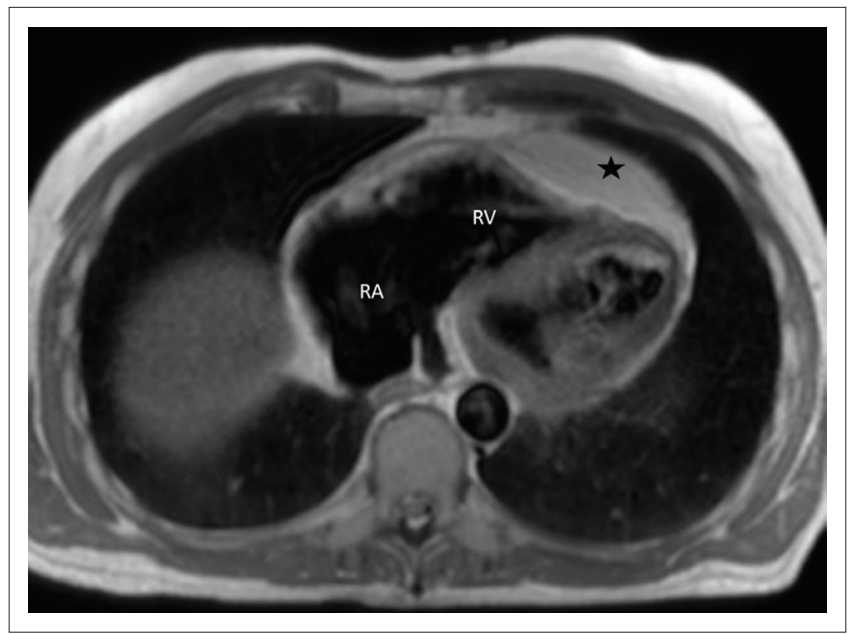

RA, right atrium; RV, right ventricle.

FIGURE 8: A 33-year-old man with liposarcoma of the pericardium. The axial black blood image demonstrates a large hyper-intense mass arising from the pericardium adjacent to the right ventricle (black star). 

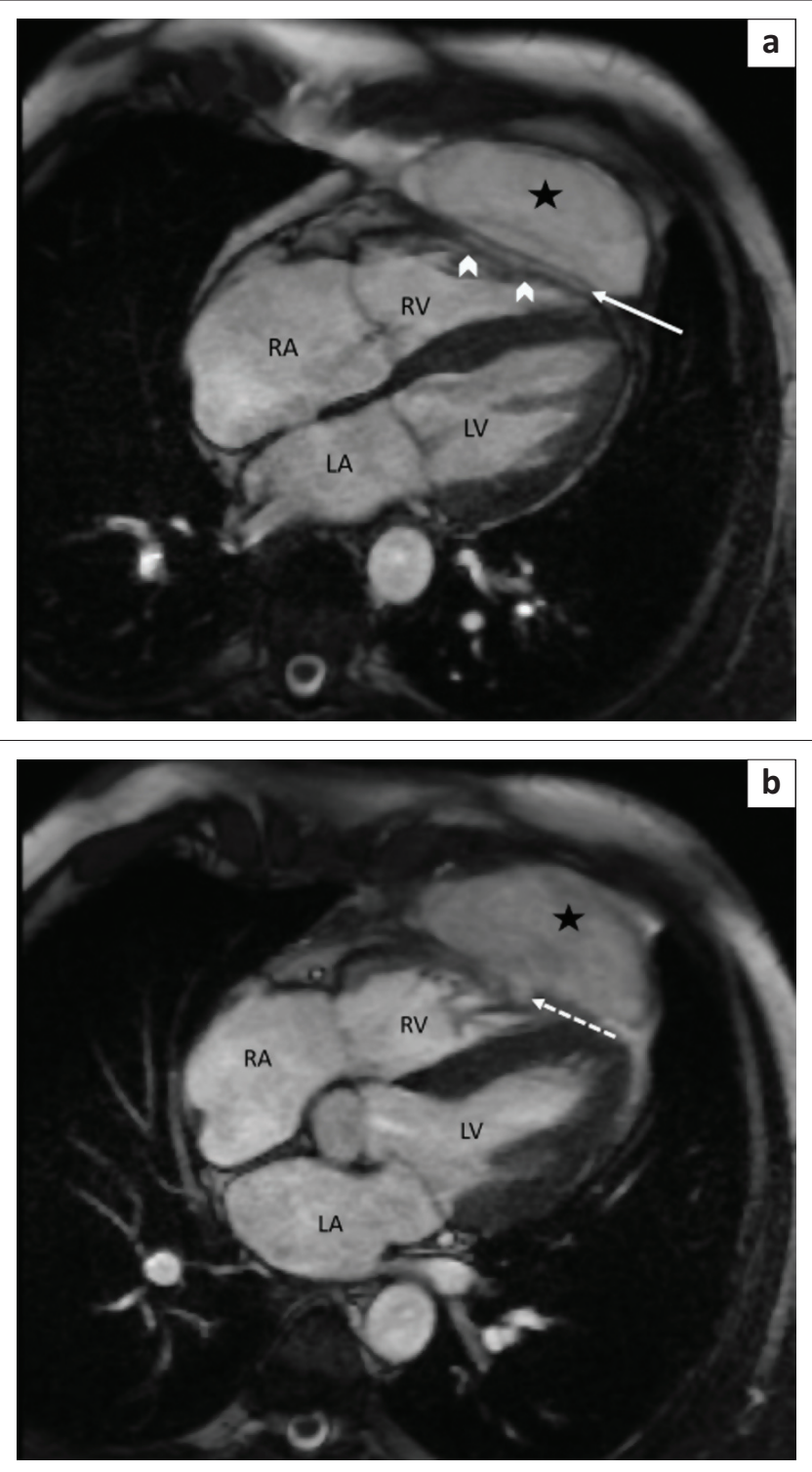

$R A$, right atrium; $R V$, right ventricle; $L A$, left atrium; $L V$, left ventricle.

FIGURE 9: A 33-year-old man with liposarcoma of the pericardium. The cine SSFP axial image (a) demonstrates a large hyper-intense mass adjacent to the right ventricle (*) with clear separation (white arrow) between the mass and the right ventricular myocardium (white arrow heads) during systole when the ventricle moves away from the lesion. The cine SSFP axial image (b) demonstrates a small area of local invasion of the right ventricular myocardium (white dashed arrow) at the mid-ventricular level with extension of the tumour into the right ventricle.

The patient underwent surgical resection of the lesion and part of the right ventricular wall. The histology demonstrated a low-grade liposarcoma.

\section{Diagnosis: Liposarcoma of the pericardium}

Primary pericardial tumours are extremely rare, with a prevalence of $0.001 \%-0.007 \% .^{10}$ Metastases and direct infiltration of the pericardium by direct extension or haematogeneous spread from lung and breast cancer are most common. The most common benign pericardial masses are pericardial cysts and pericardial lipomas. ${ }^{10}$ Mesotheliomas, sarcomas and lymphoma are the most common primary pericardial tumours. ${ }^{11}$

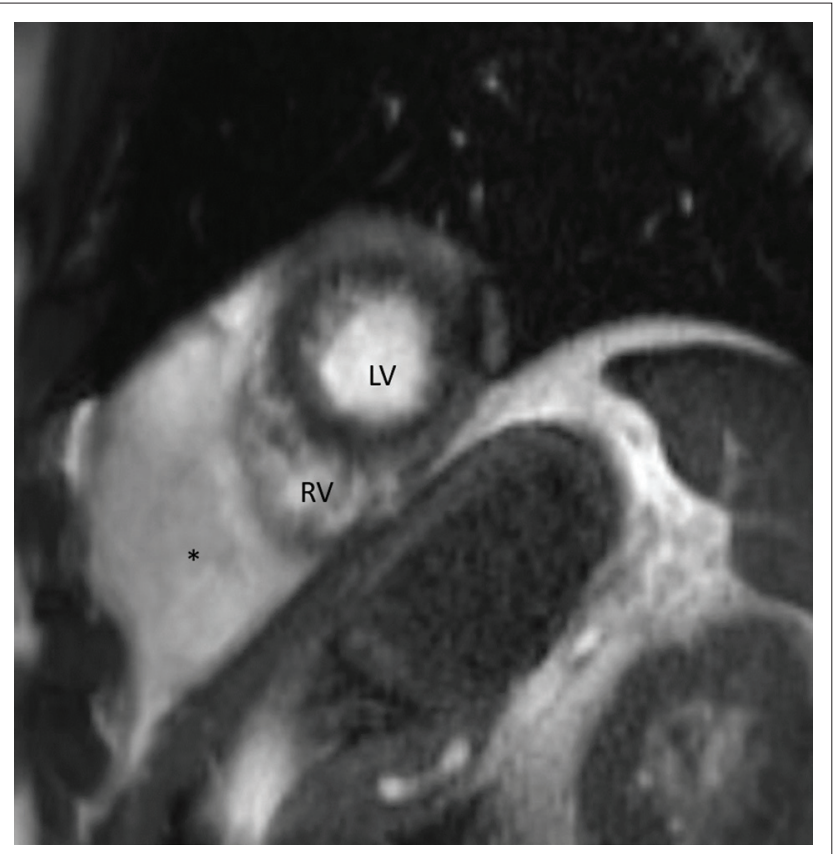

$L V$, left ventricle; RV, right ventricle.

FIGURE 10: A 33-year-old man with liposarcoma of the pericardium. The axial black blood image demonstrates a large hyper-intense mass arising from the pericardium adjacent to the right ventricle (black star).

Primary sarcoma of the pericardium is uncommon. There is a wide range of histopathological subtypes but the prognosis of these groups is uniformly poor. The mean survival for these patients ranges between 3 months and 12 months. ${ }^{10}$

Liposarcomas are normally slow growing and are usually large at the time of presentation. The late presentation often prevents complete excision of these tumours and, because of the lack of response to chemotherapy and radiation, contributes to the poor prognostic outcome. ${ }^{12}$

\section{Pericardial CMR imaging pulse sequences}

The variety of sequences provided by cardiac MRI allows the pericardium and its lesions to be visualised and characterised. However, this is also often the downfall of the modality as it is easy to become lost in the large choice of potential sequences, resulting in prolonged investigations and non-diagnostic studies. The basic sequences outlined below will provide adequate clinical information in a reasonable time for the majority of pathologies.

\section{Suggested pulse sequences}

- Anatomy and outline of the pericardium is best seen on black blood images, and it helps to run these sequences in at least two planes: axial images at a slice thickness of $8 \mathrm{~mm}$ as well as short-axis images (Figure 3) with a slice thickness of $8 \mathrm{~mm}$.

- Pericardial oedema is best demonstrated on the STIR images in a short-axis plane with a slice thickness of $10 \mathrm{~mm}$ (Figure 5).

- Pericardial enhancement is normally best seen on the late enhancement images at approximately 12-minute post 
contrast injection (Figure 4). These sequences will also demonstrate enhancement of pericardial masses.

- Cine SSFP images in a short-axis plane are useful to assess ventricular function as well as myocardial invasion (Figure 10).

- For constrictive pericarditis, real-time free-breathing images are useful to assess for septal bounce (Figures 6 and 7). This is usually done as a single slice at a mid-ventricle level. ${ }^{1}$

\section{Acknowledgements Competing interests}

The author declares that she has no financial or personal relationships which may have inappropriately influenced her in writing this article.

\section{References}

1. Bogaert J, Francone M. Cardiovascular magnetic resonance in pericardial diseases J Cardiovasc Magn Reson. 2009;11:14. http://dx.doi.org/10.1186/1532-429X-11-14

2. Rajiah P. Cardiac MRI: Part 2, pericardial diseases. AJR Am J Roentgenol. 2011 197(4):W621-W634. http://dx.doi.org/10.2214/AJR.10.7265
3. Wang ZJ, Reddy GP, Gotway MB, Yeh BM, Hetts SW, Higgins CB. CT and MR imaging of pericardial disease. Radiographics. 2003;23 Spec No:S167-80.

4. Navaneethan SD, Venkatesh S, Shrivastava R, Mehta J, Israel R. Recurrent pleura and pericardial effusions due to sarcoidosis. PLoS Med. 2005;2(3):e63. http:// dx.doi.org/10.1371/journal.pmed.0020063

5. Sekiguchi M, Yazaki Y, Isobe M, Hiroe M. Cardiac sarcoidosis: Diagnostic, prognostic, and therapeutic considerations. Cardiovasc Drugs Ther. 1996; 10(5):495-510. http://dx.doi.org/10.1007/BF00050989

6. Verhaert D, Gabriel RS, Johnston D, Lytle BW, Desai MY, Klein AL. The role of multimodality imaging in the management of pericardial disease. Circ Cardiovasc Imaging. 2010;3(3):333-343. http://dx.doi.org/10.1161/CIRCIMAGING.109.921 791

7. Kleynberg RL, Kleynberg VM, Kleynberg LM, Farahmandian D. Chronic constrictive pericarditis in association with end-stage renal disease. Int J Nephrol. 2011 2011:469602. http://dx.doi.org/10.4061/2011/469602

8. Park EA, Lee W, Kim KH, Chung JW, Park JH. Rapid progression of pericardial calcification containing a 'calcium paste' in a patient with end-stage renal disease. Circulation. 2011;123(9):e262-e264.

9. Walker CM, Chung JH, Reddy GP. Septal bounce. J Thorac Imaging. 2012;27(1):W1.

10. Restrepo CS, Vargas D, Ocazionez D, Martinez-Jimenez S, Betancourt Cuellar SL, Gutierrez FR. Primary pericardial tumors. Radiographics. 2013;33(6):1613-16S30.

11. Burazor I, Aviel-Ronen S, Imazio M, et al. Primary malignancies of the heart and pericardium. Clin Cardiol. 2014;37(9):582-588. http://dx.doi.org/10.1002/ clc. 22295

12. Lococo F, Cesario A, Meacci E, et al. Huge primary pericardial liposarcoma. Thoracic Cardiovasc Surg. 2011;59(3):172-173. http://dx.doi.org/10.1055/s-00301250187 